We review the literature on the incidence of such events and conclude that these are likely to be under-recognised and under-reported. We subsequently make a case of why these cases should be reclassified as 'never events'. Finally we make recommendations on, what procedures can be followed to minimise the incidence of such events.

\section{G411(P) CAPTURING CHILDREN AND YOUNG PEOPLE'S VOICES: IMPROVING PATIENT-CENTRED PAEDIATRIC CARE}

H L'Estrange-Snowden, S Gancarczyk, B Hopwood, J Pentelow. Patient Feedback Team, Picker Institute Europe, Oxford, UK

10.1136/archdischild-2018-rcpch.400

Aims The intention of the survey was to provide actionable insight into the experiences of children, young people and their families when receiving care as an inpatient or day case admission at NHS trusts.

Methods The Children and Young People's Inpatient Survey was developed and piloted by Picker Institute Europe alongside specialist children's hospitals in 2010. Focusing on aspects of care that children reported as important, this survey has been extended to cover day case admission and licensed to the Care Quality Commission since 2014. Running for the second time in 2017, the Children and Young People's Inpatient and Day Case Survey is now a biannual part of the National Patient Survey Programme.

This survey was specifically designed to be engaging and child-friendly, containing three versions of the questionnaire: one for parents of children aged 0-7 years; one for children aged $8-11$; and the other for $12-15$ year olds. Whilst targeted at children/young people, the last two versions contained sections for parents/guardians to also provide feedback.

Results presented are from 71 acute NHS trusts in England. Each provided a systematic stratified sample (three age brackets, date of birth and gender), totalling 73344 patients who received care between October and December 2016. Feedback was invited via postal questionnaire sent to patients' home addresses, followed by two reminders to non-responders.

Results An overall response rate of $26 \%(n=18,687)$ for eligible patients was achieved, of which $92 \%$ of children reported that overall they were (very) well looked after. Similarly $84 \%$ of parents felt that members of staff definitely provided information about their care and treatment that they could understand. However, $55 \%$ of children did not feel fully involved in decisions about their care and treatment, with 30\% reporting that they did not always understand what hospital staff said and 22\% not being able to fully discuss their worries.

Conclusions The survey provided a high level of actionable feedback, highlighting some areas of good performance, for example most children felt able to ask staff questions, as well as areas for improvement such as greater provision of emotional support.

\section{G412(P) SHARING 'BEST PRACTICE' AMONGST TRAINEES AND TRAINERS TO OPTIMISE THE QUALITY OF PAEDIATRIC TRAINING}

M Van de Vijver, P Prentice, O Wilkey, R Kainth. London School of Paediatrics, RCPCH, London, UK

10.1136/archdischild-2018-rcpch.401
Aims The Paediatric Trainee Survey is performed annually in a continuing effort to improve paediatric training across the deanery by anonymously obtaining feedback from all Paediatric trainees about their experiences in training. Every year, this survey highlights a wide variation in quality and practice in training across the deanery. Therefore, this project aims to identify the local practice which makes certain trusts excel in their training feedback and distribute this information across the deanery with the goal of ensuring equal standards and experiences.

Method Using the 2016 Paediatric Trainee Survey, we analysed the quantitative results and identified the top three performing trusts for each of the selected five survey indicators. These indicators covered the following aspects of training; Induction, Internal and external teaching opportunities, management experience and training, clinical leadership training opportunities and teamwork and morale in the department. The College Tutors and Trainee Representatives for the identified trusts were contacted with specific questions related to their indicator to obtain information about their local practice. This information was collated and distributed on the Deanery bulletin and website and at Deanery training days with feedback obtained through discussions with trainees and trainers.

Results Feedback was obtained and analysed from the top performing trusts and common themes in local practices were identified for each of the five survey indicators. These themes were named 'Best Practice' and shared amongst the trainees and trainers in the deanery.

Conclusion This project has highlighted main themes in practices in five key aspects of Paediatric training which have been shared across the deanery to improve the overall standard and experience of Paediatric Training. Subsequent work is required to obtain the same information amongst the remaining survey indicators highlighted in the annual Paediatric Trainee Survey and in distributing these practices to other deaneries in the United Kingdom.

\section{G413(P) NICU QUALITY IMPROVEMENT: MESSAGE OF THE WEEK!}

SSK Dhulipala, TJ Bibby, P Heap, S Nedungadi, K Tanney, M Parr. Neonatal Intensive Care Unit, Manchester University Hopsitals NHS, Manchester, UK

\subsection{6/archdischild-2018-rcpch.402}

Aims Critical or High Level Incidents are unfortunately a part and parcel of clinical care, which we all strive to eliminate or at very least minimise. In most units, when they occur, an incident form is submitted online by the clinical staff involved. An investigation may be carried out, and the individuals involved receive feedback. Often, incidents recur due to poor awareness of past events, as disseminating information to different clinical teams is challenging.

To enhance awareness of NICU High Level Incidents, this large neonatal intensive care unit has sought out novel ways to disseminate feedback on incidents occurring, to improve clinical practice and maximise learning from adverse events or near misses. As part of improving knowledge of HLIs and the investigation outcomes, a middle-grade Risk Management role was created in May of this year, alongside the corresponding senior nursing roles.

Methods Since commencement of this QI initiative, the named Risk registrar acts as a liaison doctor, disseminating feedback 